\title{
Potential role of ultrasound imaging in interstitial image based cervical cancer brachytherapy
}

\author{
Mitchell Kamrava, MD \\ Department of Radiation Oncology, University of California, Los Angeles, USA
}

\begin{abstract}
In 2012, more than 500,000 cases of cervical cancer were diagnosed worldwide. Over three quarters of these cases occur in less developed countries [1]. Advancements in image-guided brachytherapy are resulting in improved outcomes and reduced morbidity for women with this disease, but its worldwide adoption is hampered by lack of accessibility to advanced imaging techniques. Ultrasound is emerging as a potential option for tumor visualization, brachytherapy catheter placement, and treatment planning. While additional work is needed, ultrasound can potentially serve as the sole imaging modality for catheter insertion and planning. This paper will review our current knowledge on the use of ultrasound in interstitial brachytherapy treatment for cervical cancer.
\end{abstract}

J Contemp Brachytherapy 2014; 6, 2: 223-230 DOI: $10.5114 /$ jcb.2014.43778

Key words: brachytherapy, cervical cancer, image guided.

\section{Purpose}

Brachytherapy is an essential component in the definitive management of cervical cancer. It allows dose escalation to over $80 \mathrm{~Gy}$ in biologically equivalent dose in 2-Gy fractions, while minimizing dose to surrounding normal tissues. Outcomes data using image-guided brachytherapy (IGBT) show local control in the $90 \%$ range, when the $\mathrm{D}_{90}$ to the high-risk clinical target volume (HR-CTV) is in the high 80 Gy range [2,3]. Similar data on 592 patients enrolled on the retro-EMBRACE study showed a $D_{90}$ to the HR-CTV of $>92$ Gy resulted in an overall local control rate of $95 \%$ [4].

In order to achieve such high doses to the HR-CTV while limiting dose to normal tissues, appropriate brachytherapy applicator selection is critical. Applicator choices include intracavitary (IC), interstitial (IS), or hybrid approaches. The hybrid approach is easier to perform than an IS implant and in the Vienna IGBT series, for example, $44 \%$ of patients were treated with this method [5]. This underscores the point that standard three channel tandem and ovoids do not ideally cover the HR-CTV in a substantial percentage of cases.

For cases where hybrid type applicators cannot provide adequate target coverage, IS implant is the best treatment option. IS brachytherapy is a highly versatile technique that allows flexibility in covering residual disease well beyond what can be accomplished with IC approaches. It is unfortunately not widely utilized secondary to lack of appropriate radiation oncologist training, resources necessary to perform the implant and treat the patient (ie multiple day in-patient hospitalization), and expertise in treatment planning. These barriers however, should not detract from recognizing the merits of this technique when done properly.

Given the smaller volume of IS cases being performed, the breadth of published clinical experiences using modern 3-dimensional planning in cervical cancer is limited. The largest experience for cervical cancer patients was reported by Pinn-Bingham et al. who reported on 116 cervical cancer patients treated with 50.4 Gy external beam radiation (EBRT) to the pelvis, followed by 2 separate implants of 6 Gy $\times 3$ fractions each (total brachytherapy dose of $36 \mathrm{~Gy}$ ) [6]. The first implant was done 1-2 weeks after completing EBRT and the second was done 2 weeks after that. All patients were CT planned. About $60 \%$ of the patients also received hyperthermia and about $80 \%$ received chemotherapy. With a median follow-up of 35.1 months, clinical local regional control was achieved in about $85 \%$ of patients, while approximately $13 \%$ experienced grade 3 late toxicities. Sharma et al. reported on 42 cervical cancer patients treated with 2 weekly brachytherapy sessions of 10 Gy in combination with EBRT (40 Gy), followed by a midline block with an additional 10 Gy in 5 fractions [7]. With a median follow-up of 23 months, they reported 3 year relapse free survival rates for stage IIB, IIIB, IVA of $67 \%, 34 \%$, and $20 \%$, respectively. In another relatively large series, Kannan et al. reported on 47 patients treated with 45 Gy EBRT followed by IS brachytherapy of 3.75-5 Gy x 5 fractions [8]. Reported clinical outcomes were 2-year actuarial local control of $61 \%$, disease free survival of $43 \%$, 
Table 1. Comparison of ultrasound and MRI for interstitial gynecologic brachytherapy. One can see that the main limitation of MRI is access and that in order for ultrasound to be comparable with MRI that there are many areas that need to be improved

\begin{tabular}{lll} 
& Ultrasound & MRI \\
\hline Accessibility in the operating room & High & Low \\
\hline Real-time image guidance & High & Low \\
\hline Catheter visualization & High & High \\
\hline Target visualization & High & High \\
\hline Volume based evaluation & Low & High \\
\hline Treatment planning & Low & High \\
\hline Experience with technique & Low & High \\
\hline Clinical evidence & Low & High
\end{tabular}

and $\geq$ grade 3 toxicity of $10 \%$. Lastly, Mahantshetty et al. published their experience with IS gynecologic brachytherapy in 113 patients ( 37 with cervical cancer post-inadvertent surgery) with a median dose of $73 \mathrm{~Gy}$, and follow-up of 43 months. 3 year disease free and overall survival for the cervical cancer patients was $61 \%$ and $64 \%$, respectively. Grade III/IV rectal/bladder/small bowel toxicity was $10 \%, 4.5 \%$, and $6 \%$, respectively [9]. While this is not an exhaustive review of the totality of literature for IS IGBT for cervical cancer, it does represent some of the larger published studies. The various treatment approaches including total dose and fractionation in addition to the short follow-up make drawing conclusions and comparisons between series difficult. This is also reflected in the recent American Brachytherapy Society guidelines on cervical cancer that lists 5 different suggested IS fractionation regimens [10]. Clearly moving forward, additional data is necessary to determine the ideal dose/ fractionation and normal tissue constraints using this technique.

\section{Issues with the implementation of image guidance for IS brachytherapy}

While IC IGBT has improved outcomes and reduced morbidity, its adoption by clinics around the world is somewhat limited secondary to the resources necessary to execute MRI based applicator insertion and planning [11]. To address this, many hybrid approaches to use MRI for the first insertion and CT for the subsequent ones have emerged as possible compromises [12]. This elegant solution is not applicable for IS brachytherapy as this procedure is performed in an operating room, and very few centers in the world have access to intra-operative CT or MRI imaging [13]. Trans-abdominal ultrasound, while adequate to assess tandem placement, does not enable adequate visualization of parametrial catheters. This creates a dilemma in terms of how to ensure an appropriate catheter distribution prior to leaving the operating room where one may be placing over 10 catheters.
If one starts from the beginning to consider what the ideal imaging modality (fluoroscopy, CT, MRI, or ultrasound) for IS brachytherapy catheter insertion and treatment planning would be, there are multiple factors to consider. Van Dyk et al. presented a nice table on the pros and cons of various imaging modalities for gynecologic brachytherapy and determined the ideal imaging modality would be accessible for each insertion, provide imaging intraoperatively, and accurately visualize the applicator/catheters, residual tumor, cervix, and organs at risk [14].

CT and MRI meet these criteria, except for the fact that they are not necessarily accessible for each insertion. So, while MRI serves as the gold standard for IC based IGBT, its accessibility is limited for interstitial techniques.

Trans-rectal or trans-vaginal ultrasound can solve the issues of access that limit MRI but present other barriers in terms of experience and validation of the technique for assessing residual disease (Table 1).

\section{Use of ultrasound in the assessment of cervical cancer}

For ultrasound to serve as a platform for image guidance for IS brachytherapy, it needs to be able to accurately visualize the target, particularly after a course of chemoradiation (Figs. 1 and 2). Epstein et al. reported on a multi-institution prospective study of 182 women comparing the diagnostic accuracy of MRI and trans-rectal or trans-vaginal ultrasound in early stage cervical cancer (FIGO stages IA2-IIA1) patients with pathology at the time of surgery as a reference standard [15]. The maximum tumor diameter was not significantly different when measured by ultrasound, MRI, or histology. The volume of the tumor with each modality was not reported. Ultrasound was also significantly better than MRI in assessing parametrial involvement with a specificity of $98 \%$ versus $92 \%(p<0.001)$ as well as visualizing residual disease in patients who had a cone biopsy performed prior to surgery. This study demonstrates ultrasound as an accurate imaging modality in early stage cervical cancer, however does not provide evidence for its accuracy in locally advanced cases.

A smaller amount of data is available, validating ultrasound based measurements in the locally advanced setting as surgery is not routinely performed. In one small study, 3-dimensional trans-vaginal ultrasound and MRI was prospectively examined in 24 patients with locally advanced cervical cancer prior to surgery [16]. Overall cancer staging accuracy for clinical examination, MRI and ultrasound were: $63 \%, 41 \%$, and $67 \%$, respectively.

In Europe, neoadjuvant treatment with either chemotherapy alone or chemoradiation prior to surgery for locally advanced patients, is an emerging treatment option and provides another opportunity to compare ultrasound and MRI with final pathology. One prospective study of 30 patients with locally advanced cervical cancer treated with neoadjuvant chemotherapy followed by surgery, compared tumor measurements between trans-rectal ultrasound and MRI [17]. There was significant agreement in both intraclass and interclass correla- 

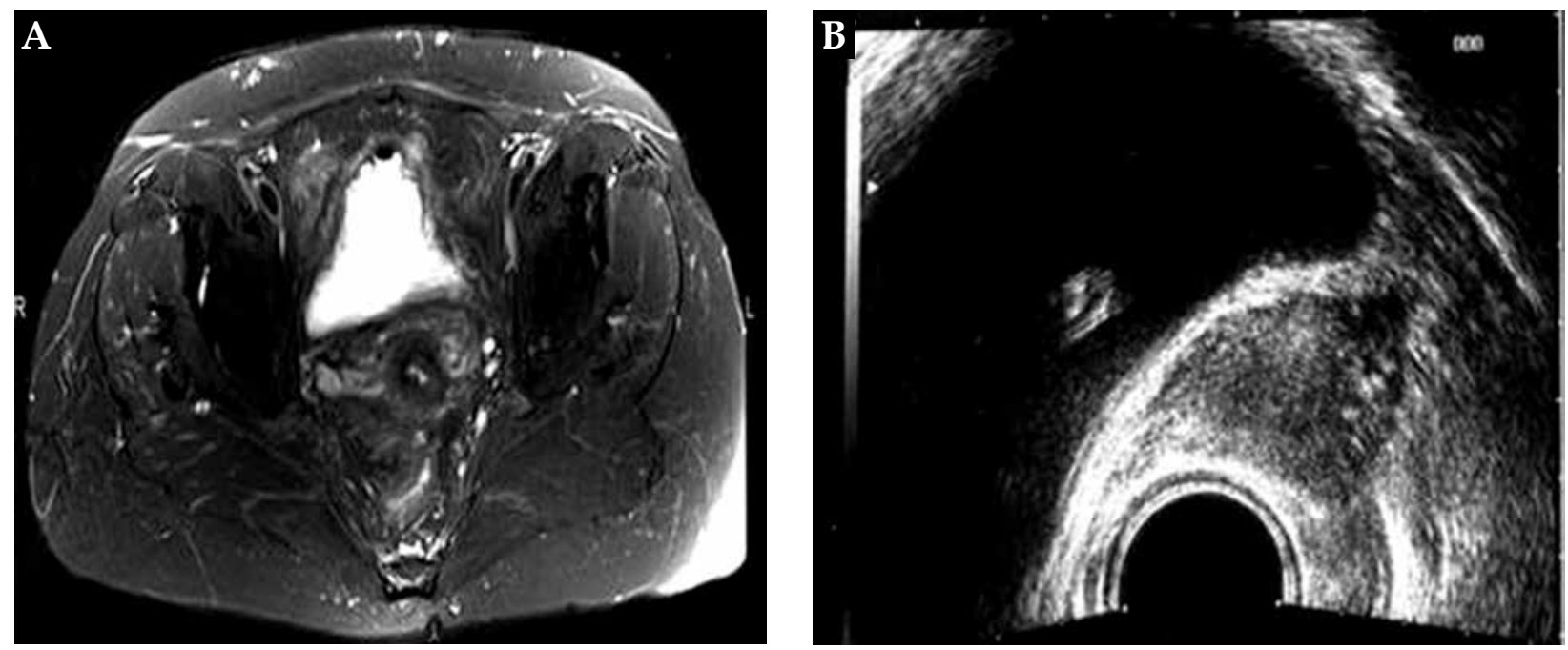

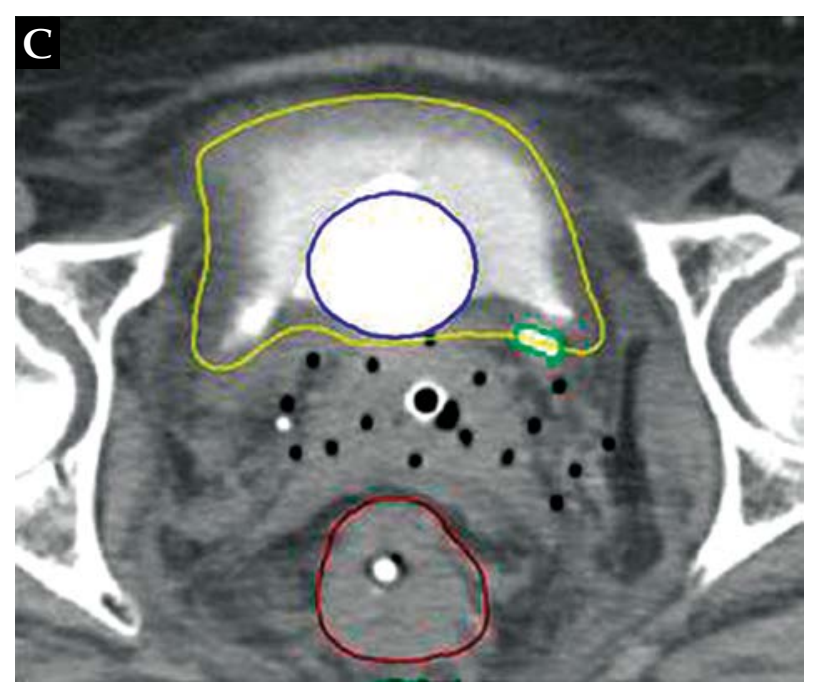

tion between tumor volume estimates from ultrasound and histology, but not so with MRI and histology. After neoadjuvant chemotherapy, the maximum tumor diameter $(\mathrm{mm})$ / volume $\left(\mathrm{mm}^{3}\right)$ on MRI, trans-rectal ultrasound, and histology was 18.6/2888, 16.2/2886, and 15.9/2106, respectively. The larger volumes seen on MRI and ultrasound were likely secondary to shrinkage issues during the processing of the specimen after surgery. The overall accuracy of detecting non-microscopic residual tumor $\left(>5 \mathrm{~mm}^{3}\right)$ was $77 \%$ for both MRI and ultrasound.

Other studies have looked at the accuracy of ultrasound after chemoradiation. Testa et al. reported on a prospective study on a total of 42 patients with FIGO stages IB2, IIA with tumor size $\geq 4 \mathrm{~cm}$, IIB, III, and IV treated with neoadjuvant treatment prior to surgery [18]. Trans-vaginal ultrasound and MRI tumor measurements 1 week prior to surgery were compared with final pathology at the time of surgery. 22 of these patients were treated with neoadjuvant chemoradiation (39.6 Gy to the whole pelvis with concurrent cisplatin/5-fluorouracil). Agreement on the presence of cervical tumor at the time of surgery was $88 \%$ between ultrasound and MRI. Ultrasound yielded four false-negative and four
Fig. 1. Example of T2 weighted MRI (A), ultrasound (B), and CT (C) of a FIGO stage IIB squamous cell cervical cancer images obtained following 45 Gy external beam radiation therapy with concurrent cisplatin chemotherapy. Similarities can be seen in the axial dimensions of the residual disease/cervix on all three imaging modalities

false-positive cases, while MRI yielded seven false-negative cases and three false-positive cases. All four patients with false negative ultrasound results received neoadjuvant treatment. Microscopic disease was seen in three of four cases, and in one case there was residual disease involving $0.7 / 1 \mathrm{~cm}$ of the cervical stroma. In the false positive cases, histopathology revealed only fibrosis and reactive tissue. While this study is small, it sets the precedent that ultrasound after neoadjuvant therapy either with chemotherapy or chemoradiation can provide an accurate assessment of residual tumor that is similar to MRI. More importantly, in the cases where ultrasound misclassified the extent of residual disease, the misclassification would not have impacted the patient's treatment if the ultrasound based volume was used for brachytherapy catheter insertion and planning, as the entire cervix is contoured as part of the HR-CTV.

Schmid et al. also recently reported on trans-rectal ultrasound and it's correlation with MRI imaging [19]. A total of 17 patients with cervical cancer were studied. The study showed close correlation between the two imaging modalities, with a maximum target width/thickness of $4.2 / 3.3 \mathrm{~cm}$ versus $4.2 / 3.1 \mathrm{~cm}$ for MRI and TRUS. 

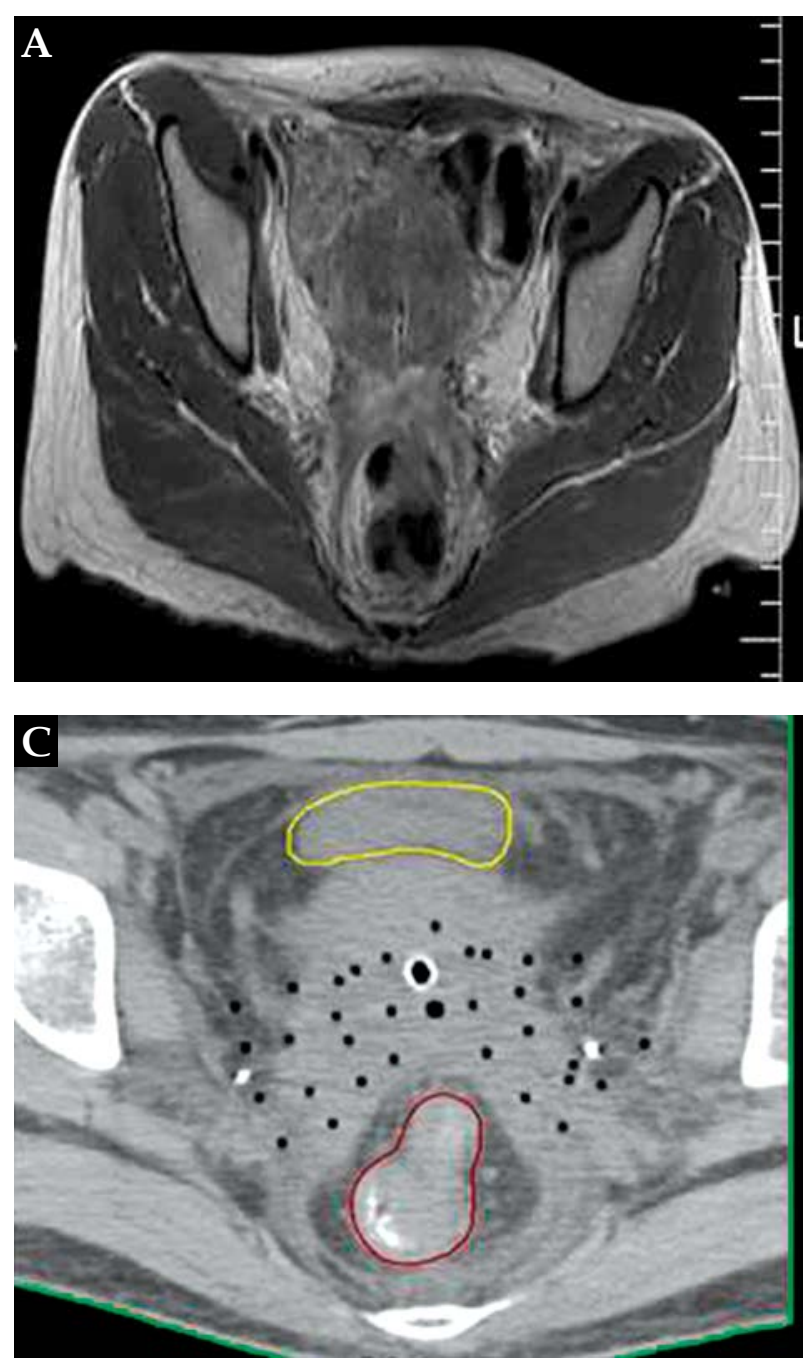

These studies are small, but demonstrate similar extents of disease on ultrasound and MRI. Ultimately larger series are needed to confirm these findings. Lastly, to move ultrasound based target definition forward, there needs to be a study that demonstrates concordance between HR-CTV volumes determined on ultrasound and MRI. If validated, this would allow an easy transfer of contouring definitions from the MRI based GEC-ESTRO guidelines to ultrasound based ones.

\section{Use of ultrasound in placement of brachytherapy catheters for IS gynecologic brachytherapy}

There are early reports of using ultrasound for gynecologic brachytherapy dating back to the 1970s [20-22] One of the first reported uses of ultrasound for IS gynecologic brachytherapy was reported by Stock et al. who demonstrated the usefulness of ultrasound to visualize the target as well as normal tissues in 12 patients [23]. Sharma et al. reported on their experience using TRUS to assist with needle placement in 40 procedures performed in 25 patients [24]. No needles in the bladder, rectum or uterine perforations were noted with this technique. The average duration of the implant procedure was

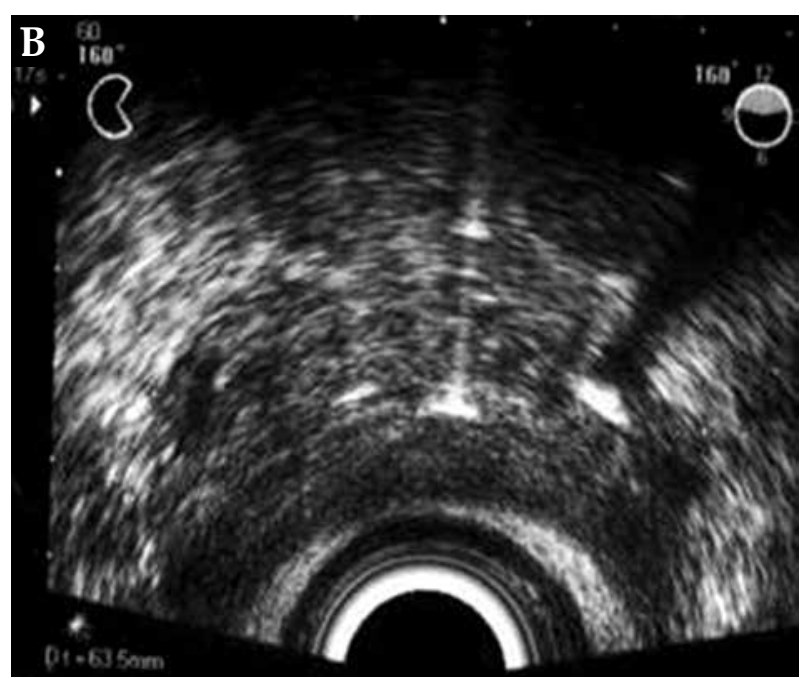

Fig. 2. Example of T2 weighted MRI (A), ultrasound (B), and CT (C) of a FIGO stage IIIB squamous cell cervical cancer images obtained following 45 Gy external beam radiation therapy with concurrent cisplatin chemotherapy. Similarities can be seen in the axial dimensions of the residual disease/cervix, including involvement of the uterosacral ligaments bilaterally, on all three imaging modalities

50 minutes. Other groups have also reported experiences using ultrasound to assist the guidance of brachytherapy catheters with each demonstrating feasibility and safety, but all recognizing the learning curve associated with mastery of the anatomy and identification of catheters.

\section{Institutional IS brachytherapy insertion technique with ultrasound}

Once a decision has been made to proceed with interstitial brachytherapy, there are many aspects to consider in the operating room to ensure a successful implant. Following the induction of anesthesia, one must consider the ideal patient set-up. Patients are placed in the dorsal lithotomy position, however one needs to decide on the type of stirrups used. Yellow-fin stirrups are easier to use, but if one plans on using cross-table lateral fluoroscopy it could create a problem, as one of the rubber pieces of the yellow-fins typically blocks the lateral fluoroscopy beam and creates a black spot obscuring the $X$-ray image over the catheters. Candy-cane stirrups do not create this difficulty, and they have been our choice for these procedures. As one develops more experience with ultrasound based catheter insertion, the need to 

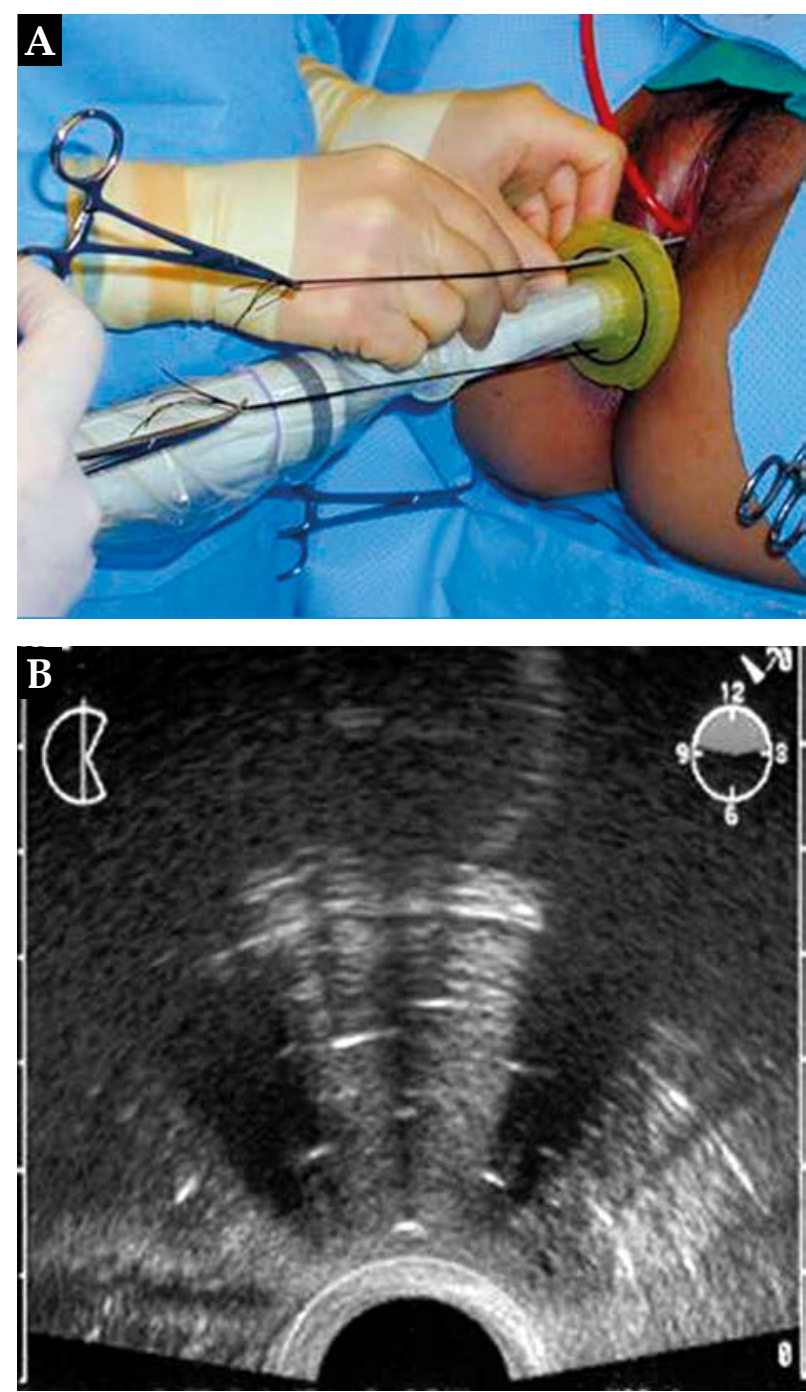

double check one's catheter distribution with fluoroscopy becomes obsolete.

Once the patient is appropriately positioned, one should evaluate and understand the patient's anatomy and extent of residual disease both on physical examination as well as on ultrasound. When utilizing the ultrasound, one should be aware of whether one is using an end fire or a side fire probe. One of the main differences one will notice when using trans-rectal ultrasound in these gynecologic cases, is that depending on whether the probe is end fire or side fire, one may not be able to appreciate the full extent of the uterus (Fig. 3). This poses a problem when trying to visualize the placement of the tandem up to the top of the fundus. Some have circumvented this issue by using a trans-abdominal ultrasound to confirm appropriate tandem placement.

After understanding the anatomy, it is helpful to place seeds to mark the residual extent of disease. This is helpful for contouring if planning on CT imaging, as well as on fluoroscopy if one needs to check the relative positioning of catheters. Visualization of gold markers is possible on MRI and appears as flow voids, but they are not very distinct.

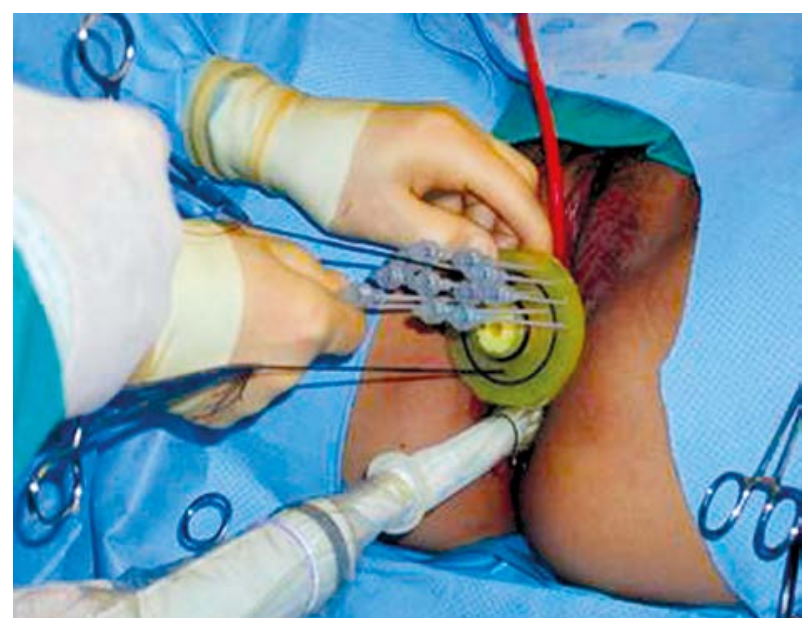

Fig. 3. Panel A demonstrates the ultrasound being placed through the central hole in the template, which is typically where the cylinder is placed. Placing the ultrasound through this hole allows improved visualization of the anterior catheters, especially if one is trying to place periurethral catheters. One can also see the technique of pulling on the countertraction sutures to assist with catheter placement. On the right one can see placement of the trans-rectal ultrasound for assistance with the placement of catheters posteriorly. In Panel B one can see an example of an axial ultrasound image with the bright dots representing the flexi-guides

After this step countertraction sutures should be placed in the cervix. These will assist in holding the template stable during catheter insertion. After the procedure is completed it will also help to stabilize the template. After determining the extent of residual disease, one can determine roughly which holes in the perineal template will be needed to encompass the disease. The ability to see the target in real time eliminates the need to preplan the catheter distribution. If the full extent of the template is not going to be used, it is helpful to use a 20 blade to cut it down to the size that will be needed. This is useful because it leaves a less bulky apparatus between the patient's legs, making it more comfortable during the remainder of her in-patient stay. Prior to placing the template on the perineum, one should sound and dilate the cervical OS under ultrasound guidance. The cervical OS typically needs to be dilated to a size 6 Hegar dilator prior to the central tandem being able to comfortably fit. After the template has been prepared, it is placed against the perineum and the countertraction sutures should be pulled through it. Typically, one can use the holes at the 12:00 and 6:00 positions, as these are not used for inserting catheters. 

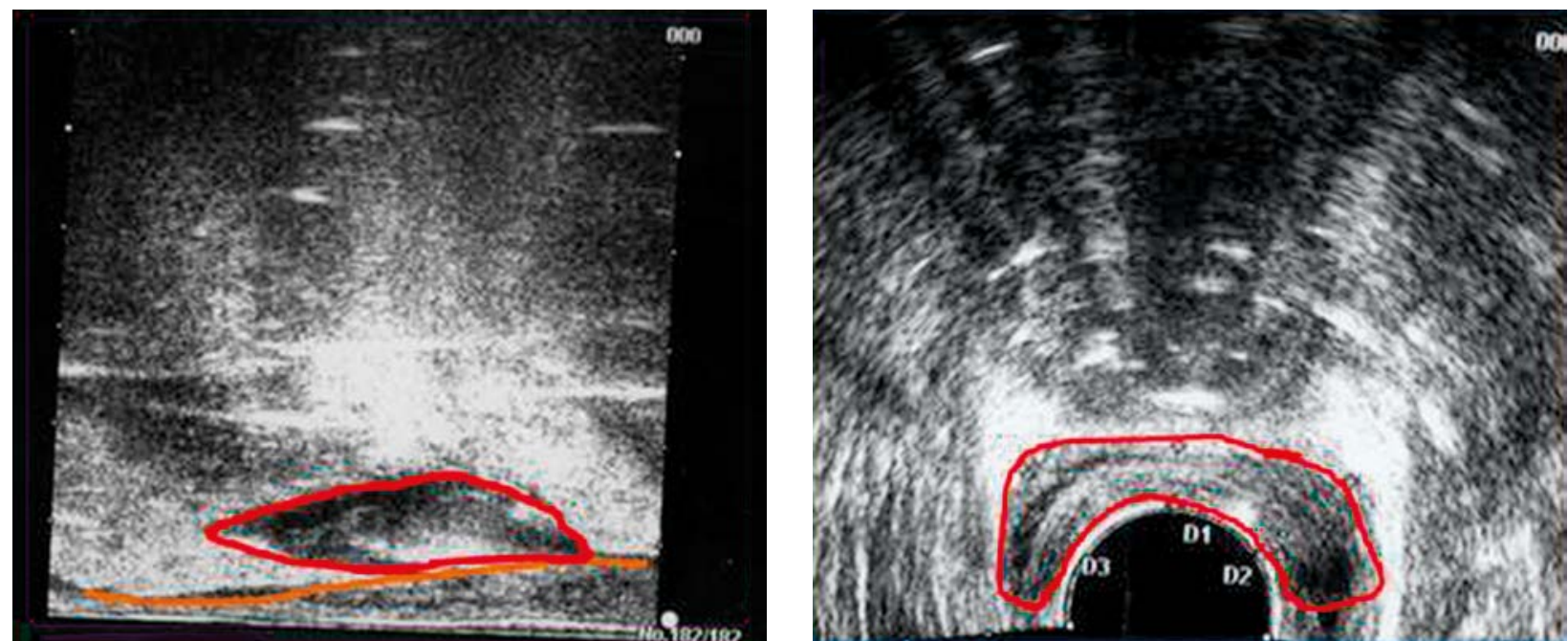

Fig. 4. An example of a sagittal (left panel) and axial (right panel) ultrasound image of the space created between the posterior vaginal wall and anterior rectum after hydrodissection. The space that's been opened up during this process is outlined in red.

The rectal wall is outlined in brown

With the template, tandem, and central cylinder in place, one can insert the TRUS and hold the countertraction sutures for stability, while another person starts to place the flexi-guides. It is easiest to start with placing the flexi-guides through the grooves of the central cylinder at the 11:00 and 2:00 positions, followed by the 7:00 and 5:00 positions. After placement of the central catheters, one can build the remainder of the implant. It is typically easiest to place the most anterior catheters and to subsequently place the more posterior rows. Until one is facile with ultrasound, fluoroscopy can be used intermittently in both the AP and lateral directions to confirm the catheter distribution. At the end of the case, a bolster can be placed around the edges of the template to make it more comfortable for the patient, as well as to stabilize it.

Theoretically, if ultrasound based volumes are concordant with MRI a CT simulation should be adequate for 3-dimensional planning. One should be able to contour around the brachytherapy catheters as they would have been placed on the periphery of the ultrasound based target, thereby helping to correct for the over-contouring of the cervix that is seen when using CT based planning [25].This is a hypothesis that has not been validated clinically and results using this approach are needed to validate this work-flow.

\section{Radiation treatment planning with ultrasound}

Ultimately, the ideal treatment process following ultrasound based catheter insertion would be to perform real-time ultrasound planning. One could imagine the patient being treated and the implant being removed prior to the patient waking up or possibly delivering two treatments in one day and having the patient go home. The only disadvantage of this approach is that it would require the patient to have multiple separate implants as opposed to one implant with multiple fractions given as an in-patient. Which is preferable to the patient is debat- able, although a recent study suggests that an overnight stay can lead to significant stress [26].

There is limited data using ultrasound based planning for gynecologic brachytherapy. The largest experience with this technique is from the Peter MacCallum Cancer Center for intracavitary based brachytherapy [14]. This is a "2.5-dimensional" approach in that the ultrasound measurements are used to inform the planning, however the prescription is not based on a 3-dimensional volume. They reported similar target coverage, rectal point, and vaginal mucosa dose between trans-abdominal ultrasound and MRI based plans delivered to a volume. Early clinical outcomes showed $90 \%$ local control and late RTOG bowel toxicity (Grade 3/4) of $<2 \%$.

One of the great limitations of moving forward with ultrasound based planning is the need to be able to create 3-dimensional volumes of the cervix and residual disease. 3-dimensional ultrasound is now available and commonly used in obstetrics, but is not widely adopted for the assessment of gynecologic malignancies [27]. It allows imaging through any plane through a region of interest. This volume can be acquired and stored allowing further analysis such as virtual navigation, multiplanar display, and surface rendering. Clinical data using 3-dimensional ultrasound in cervical cancer is limited [27]. Even if 3-dimensional volumes could be obtained, there are still issues with treatment planning that need to be addressed. Some of this has been solved for high-dose-rate prostate brachytherapy, however further work is clearly needed if ultrasound is going to be used for more than just catheter guidance [28].

\section{Emerging applications of real-time ultrasound}

One of the disadvantages of CT or MRI for interstitial brachytherapy is that it requires multiple interations of placing catheters, stepping out of the room, taking an image, reviewing the image, making adjustments, taking more images, and then repeating this until the implant 
is complete. Ultrasound is different in that it allows real time visualization of the catheters. This permits for some additional procedures to be done that would be more challenging without real time guidance. For example, using real-time guidance to create space between the posterior vaginal wall and anterior rectum. The rationale for this would be to improve the potential therapeutic ratio of brachytherapy, as one of the serious potential side effects of brachytherapy is the development of a recto-vaginal fistula.

This technique has been introduced in prostate cancer with the use of products such as SpaceOAR [29]. For prostate cancer, this has been straight forward as only about $10 \mathrm{cc}$ of material is needed to create approximately a centimeter of separation. Figure 4 shows an example of an axial ultrasound image showing the potential space that can be created between the posterior vaginal wall and anterior rectum. Reports of utilizing this technique for women with gynecologic cancers have been reported with varying success in the recurrent as well as definitive settings [30-32]. Additional work in this area is needed to determine whether this separation will have any clinical significance.

\section{Conclusions}

Trans-rectal or trans-vaginal ultrasound is emerging as a highly accessible and cost effective method to perform IS cervical cancer brachytherapy. Development of ultrasound based real-time treatment planning for gynecologic brachytherapy needs further development. Integration of 3-dimensional ultrasound to allow volume based planning needs to be investigated. Advances in ultrasound based catheter insertion and planning will allow greater access to image guided brachytherapy for challenging cases that are not suitable for IC applicators.

\section{Disclosure}

Speaking Honorarium from Elekta in 2014.

\section{References}

1. Globocan 2013. Cervical Cancer Estimated Incidence, Mortality and Prevalence Worldwide in 2012. Available at: http:/ / globocan.iarc.fr/Pages/fact_sheets_cancer.aspx. Last accessed February 2014

2. Dimopoulos JC, Lang S, Kirisits C et al. Dose-volume histogram parameters and local tumor control in magnetic resonance image-guided cervical cancer brachytherapy. Int J Radiat Oncol Biol Phys 2009; 75: 56-63.

3. Dimopoulos JC, Land S, Potter R et al. Dose-effect relationship for local control of cervical cancer by magnetic resonance image-guided brachytherapy. Radiother Oncol 2009; 93: 311-315.

4. Tanderup K, Fokdal L, Sturdza A et al. Dose-response for local control in image guided cervix brachytherapy in the retroEMBRACE study. Presented at ESTRO 2013. http://www. estro.org/search?q=retroEMBRACE\&alloptions $=$ on

5. Pötter R, Georg P, Dimopoulos JC et al. Clinical outcome of protocol based image (MRI) guided adaptive brachytherapy combined with 3D conformal radiotherapy with or without chemotherapy in patients with locally advanced cervical cancer. Radiother Oncol 2011; 100: 116-123.
6. Pinn-Bingham M, Puthawala AA, Syed AM et al. Outcomes of high-dose-rate interstitial brachytherapy in the treatment of locally advanced cervical cancer: long-term results. Int J Radiat Oncol Biol Phys 2013; 85: 714-720.

7. Sharma DN,Rath GK, Thulkar S et al. High-dose rate interstitial brachytherapy using two weekly sessions of $10 \mathrm{~Gy}$ each for patients with locally advanced cervical carcinoma. Brachytherapy 2011; 10: 242-248.

8. Kannan N, Beriwal S, Kim H et al. High-dose-rate interstitial computed tomography-based brachytherapy for the treatment of cervical cancer: early results. Brachytherapy 2012; 11: 408-412.

9. Manhantshetty U, Shrivastava S, Kalyani W et al. Templatebased high-dose-rate interstitial brachytheapy in gynaecological cancers. Brachytherapy 2013; S1538-4721.

10. Viswanathan AN, Beriwal S, De Los Santos JF et al. American Brachytherapy Society consensus guidelines for locally advanced carcinoma of the cervix. Part II: high-dose-rate brachytherapy. Brachytherapy 2012; 11: 47-52.

11. Tharavichitkul E, Wanwilairat $S$, Chakrabandhu $S$ et al. Image-guided brachytherapy (IGBT) combined with whole pelvic intensity-modulated radiotherapy (WP-IMRT) for locally advanced cervix cancer: a prospective study from Chaing Mai University Hospital Thailand. J Contemp Brachytherapy 2013; 5: 10-16.

12. Beriwal S, Kannan N, Kim H et al. Three-dimensional high dose rate intracavitary image-guided brachytherapy for the treatment of cervical cancer using a hybrid magnetic resonance imaging/computed tomography approach: feasibility and early results. Clin Oncol 2011; 23: 685-690.

13. Viswanathan AN, Szymonifka J, Tempany-Afdhal CM et al. A prospective trial of real-time magnetic resonance-guided catheter placement in interstitial gynecologic brachytherapy. Brachytherapy 2013; 12: 240-247.

14. Van Dyk S, Narayan K, Fisher R et al. Conformal brachytherapy planning for cervical cancer using transabdominal ultrasound. Int J Radiat Oncol Biol Phys 2009; 75: 64-70.

15. Epstein E, Testa A, Gaurilcikas A et al. Early stage cervical cancer: tumour delineation by magnetic resonance imaging and ultrasound - a European multicentre trial. Gynecol Oncol 2013; 128: 449-453.

16. Byun J, Kim Y, Jeong D et al. Three dimensional transvaginal ultrasonography for locally advanced cervical cancer. Int J Gynecol Cancer 2013; 23: 1459-1464.

17. Pinkavova I, Fischerava D, Zikan Met al. Transrectal ultrasound and magnetic resonance imaging in the evaluation of tumour size following neoadjuvant chemotherapy for locally advanced cervical cancer. Ultrasound Obstet Gynaecol 2013; 42: 705-712.

18. Testa A, Ludosvisis M, Manfredi R et al. Transvaginal ultrasonography and magnetic resonance imaging for assessment of presence, size and extent of invasive cervical cancer. Ultrasound Obstet Gynecol 2009; 34: 335-344.

19. Schmid MP, Potter R, Brader P et al. Feasibility of transrectal ultrasonography for assessment of cervical cancer. Strahlenther Onkol 2013; 189: 123-128.

20. Weitmann HD, Knocke TH, Waldhäusl C et al. Ultrasoundguided interstitial brachytherapy in the treatment of advanced vaginal recurrences from cervical and endometrial carcinoma. Strahlenther Onkol 2006; 182: 86-95.

21. Wenzel W. Improved radiotherapy dose calculations using ultrasound uterine localization. I Clin Ultrasound 1975; 3: 311-312.

22. Brascho DJ, Kim RY, Wilson EE. Use of ultrasonography in planning intracavitary radiotherapy of endometrial carcinoma. Radiology 1978; 129: 163-167.

23. Stock RG, Chan $K$, Terk $M$ et al. A new technique for performing Syed-Neblett template interstitial implants for gyne- 
cologic malignancies using transrectal-ultrasound guidance. Int J Radiat Oncol Biol Phys 1997; 37: 819-825.

24. Sharma DN, Rath GK, Thulkar S et al. Use of transrectal ultrasound for high dose rate interstitial brachytherapy for patients of carcinoma of uterine cervix. J Gynecol Oncol 2010; 21: 12-17.

25. Viswanathan A, Dimopulos J, Kirisits C et al. Computed tomography versus magnetic resonance imaging-based contouring in cervical cancer brachytherapy: results of a prospective trial and preliminary guidelines for standardized contours. Int J Radiat Oncol Phys 2007; 68: 491-498.

26. Kirchheiner K, Czajka-Pepl A, Ponocny-Seliger E et al. Post traumatic stress disorder after High Dose Rate brachytherapy for cervical cancer with 2 fractions in 1 application under spinal/epidural anesthesia: incidence and risk factors. Int J Radiat Phys Oncol 2014; 89: 260-267.

27. Alcazar JL, Jurado M. Three dimensional ultrasound for assessing women with gynaecological cancer: a systematic review. Gynecol Oncol 2011; 120: 340-346.

28. Batchelar, D, Gaztanaga M, Scmid M et al. Validation study of ultrasound-based high-dose-rate prostate brachytherapy planning compared with CT-based planning. Brachytherapy 2014; 13: 75-79.

29. Pinkawa M, Corral NE, Caffaro M et al. Application of a spacer gel to optimize three-dimensional conformal and intensity modulated radiotherapy for prostate cancer. Radiother Oncol 2011; 100: 436-441.

30. Viswanathan AN, Damato AL, Nguyen PL. Novel Use of a Hydrogel Spacer Permits Reirradiation in Otherwise Incurable Recurrent Gynecologic Cancers. J Clin Oncol 2013; 31: e446-e447.

31. Kishi K, Mabuchi Y, Sonomura T et al. Eradicative brachytherapy with hyaluronate gel injection into pararectal space in treatment of bulky vaginal stump recurrence of uterine cancer. J Radiat Res 2012; 53: 601-607.

32. Marnitz S, Budach V, Weiber F et al. Rectum separation in patients with cervical cancer for treatment planning in primary chemo-radiation. Radiation Oncology 2012; 7: 109. 